\title{
WARUM und/oder WIE - Unterschiedliche psychoanalytische Konzepte weiblicher Homosexualität
}

\author{
Almut Rudolf-Petersen (Hamburg)
}

Zusammenfassung: Anders als in den Vereinigten Staaten sind im deutschsprachigen Raum seit dem Erscheinen des pionierhaften Sammelbandes Stumme Liebe (1993) kaum Arbeiten zu weiblicher Homosexualität herausgekommen. Immerhin sind einige Artikel dieses inzwischen schon über 20 Jahre alten Readers weiterhin aktuell, z. B. der von Poluda-Korte, den ich zu Beginn der vorliegenden Arbeit vorstelle: Die Autorin entwickelt das Konzept des lesbischen Komplexes, mit dessen Hilfe sie eine lesbische wie auch eine heterosexuelle Entwicklung als Weichenstellung in einem unbewussten Konflikt versteht. Sie folgt der Frage nach der Psychogenese sexueller Orientierung, also der Frage nach dem Warum von Homosexualität bzw. Heterosexualität.

Eben diese Frage hält Ermann (2009), rückblickend auf jahrzehntelange kontroverse Diskussion und Forschung, für z. Z. nicht beantwortbar. Er betrachtet es als ergiebiger, nicht von der Warum-, sondern von der Wie-Frage auszugehen, sich also mit den Grundkonflikten und spezifischen Entwicklungsaufgaben einer eigenständigen homosexuellen Entwicklung zu befassen, die bereits pränatal angelegt sein kann, den basic facts of homosexual life. Es erscheint lohnend, Ermanns die männliche Homosexualität betreffenden Überlegungen auch für weibliche Homosexualitäten zu berücksichtigen. Denn deren auffällige Variationsbreite spricht dafür, verschiedene Konzepte zu verwenden, fallweise nur das eine (das der pränatalen Bedingtheit) oder das andere (das der psychischen Weichenstellung), in anderen Fällen beide zugleich und gleichermassen.

Schlüsselwörter: Weibliche Homosexualität, lesbischer Komplex, basic facts of homosexual life

\section{Einleitung}

Im Januar 2011 lud das Institut für Psychoanalyse und Psychotherapie im Rheinland zu einem Symposium Homosexualität und psychoanalytische Ausbildung nach Köln ein - meines Wissens die erste Veranstaltung in Deutschland zu diesem Thema. Aus psychoanalytischen Lehrbüchern waren zwar in den Jahren zuvor 
Textpassagen verschwunden, die Homosexualität pathologisierten, ${ }^{1}$ und auch die Aufnahme-Praxis wird inzwischen an vielen Instituten liberaler gehandhabt, sodass heute offen schwule und lesbische Bewerber*innen bei persönlicher Eignung die psychoanalytische Ausbildung beginnen können. Aber hatte sich darüber hinaus wirklich etwas verändert? War nicht eine explizite Stellungnahme und Revision der jahrzehntelangen Haltung der psychoanalytischen Fachgesellschaften überfällig?² Die angekündigte Tagung liess erwarten, dass die im Sande verlaufende Diskussion über das komplexe Thema Homosexualität in der Psychoanalyse Fahrt aufnehmen würde.

Ich reiste guter Dinge nach Köln - und fiel aus allen Wolken, als die Stimmung in den Arbeitsgruppen und Vorträgen teilweise angespannt, fast aufgeladen war. Erst nach einer Weile verstand ich, dass dieses Symposium auch eine Art Container-Funktion zu erfüllen hatte: Freude über das Voranschreiten der Diskussion war sicherlich angebracht, aber gleichzeitig nachrangig; erst einmal musste die Empörung der vielen anwesenden homosexuellen Kolleg*innen - ich hatte mich manchmal schon gefragt, ob es in Wirklichkeit überhaupt homosexuelle Analytiker*innen gibt: ja, es gibt sie-über die jahrzehntelange Pathologisierung und den ebenso langen Ausschluss von der psychoanalytischen Ausbildung zur Sprache kommen. ${ }^{3}$ Und auch die psychische Belastung. Besonders in Erinnerung bleibt mir ein langjährig praktizierender Kollege, der seine psychoanalytische Ausbildung sozusagen incognito gemacht hatte und erzählte, er komme immer noch nicht gegen die eigene irrationale, aber furchtbar festsitzende innere Überzeugung an, ein, weil homosexuell, zweitklassiger Analytiker zu sein. ${ }^{4}$

Es gab aber noch eine andere Irritation auf dieser sehr fruchtbaren und aufregenden Tagung. Zwei Hauptredner*innen waren geladen: Michael Ermann und Eva Poluda-Korte, die ihr Konzept des Lesbischen Komplexes als Basis der Weiblichkeit erläuterte. Poluda-Korte kam beim Auditorium nicht sehr gut an; sie habe einen klassischen psychoanalytischen Vortrag gehalten, so die genervten Reaktionen, der keine neuen Erkenntnisse zu normalen lesbischen Entwicklungen erbringe. Ganz anders dann die Aufnahme des Vortrags von Ermann, der für das Konzept einer spezifischen männlich-homosexuellen Entwicklung mit bestimmten Weichenstellungen, wie ich gleich beschreiben werde, plädierte. Ich begriff jetzt: Die Kölner Tagungsgruppe brachte zwei unterschiedliche Diskurslinien zu Psychoanalyse und Homosexualität zusammen:

Für die eine Linie steht beispielhaft Poluda-Korte (1993) mit ihrem Konzept des lesbischen Komplexes. Es fusst auf Freuds Auffassung von der bisexuellen Konstitution der menschlichen Seele. Danach sind Homo- wie Heterosexualität 
gleichberechtigte Spielarten psychosexueller Entwicklung. Poluda-Korte versteht Bisexualität sowohl im Sinne von Spielraum bei der Objektwahl (S. 73) als auch im Sinne von gleichzeitig vorhandener Maskulinität bzw. Femininität (S. 87), oder anders formuliert: im Sinne der Gleichzeitigkeit von (triebtheoretisch gedacht) passiven oder aktiven Triebzielen, die dann in einem zweiten Schritt mit männlich und weiblich assoziiert werden können (Gsell \& Zürcher, 2011, S. 699).

Ermann vertritt die andere Diskurslinie: Darin geht es um die Konzeptualisierung homosexuell-psychosexueller Entwicklungen einschliesslich der Lebenswege von homosexuellen Frauen und Männern. Die dieser Linie folgenden Autor*innen halten die Frage nach den Ursachen einer sexuellen Orientierung - hetero- oder homosexuell - für zur Zeit nicht beantwortbar, jedenfalls nicht mit dem Instrumentarium der Psychoanalyse, und wenden sich der in ihren Augen ergiebigeren Fragestellung nach dem Wie homosexueller Entwicklungen zu. Ganz offensichtlich folgten die Tagungsteilnehmer*innen mehrheitlich dieser Linie, vermutlich, weil lange Zeit die Wie-Frage nicht gestellt, vielmehr vernachlässigt worden war. Ausserdem ist es nicht einfach, die WarumFrage im Hinblick auf Homosexualität zu stellen, ohne deren jahrzehntelange Pathologisierung mitzutransportieren. Ich hatte darüber hinaus den Eindruck, dass die Wie-Frage auch wegen ihrer grösseren Anschaulichkeit ${ }^{5}$ und klinischen Relevanz die Herangehensweise der Wahl beim Tagungspublikum war.

Soweit ich sehen kann, geht die von der Wie-Frage bestimmte Diskurslinie auf Isay (1990) zurück, der mit seinem jahrelangen Engagement zum hart umkämpften Antidiskriminierungsbeschluss der American Psychoanalytic Association im Jahre 1991/92 beigetragen hatte: In dessen Folge wurden 1992/93 die ersten offen homosexuellen Kandidat*innen zur psychoanalytischen Ausbildung zugelassen, und dies führte in den Jahren darauf zu einer Vielzahl von Veröffentlichungen zu homosexuellen Lebenswegen. ${ }^{6}$ Die grössere Akzeptanz des Wie-Diskurses auf der Tagung erklärt sich vermutlich auch vor diesem historisch-politischen Hintergrund.

Die Tagung hat mich noch geraume Zeit sehr beschäftigt, nicht zuletzt wegen der harten Konfrontation zwischen den sehr unterschiedlichen, fast konträren Standpunkten. Aber schliessen sich diese beiden Herangehensweisen, die Poluda-Korte und Ermann vertreten, tatsächlich aus? Wäre es nicht möglich, dass sich die Divergenz der Konzepte auch aus der Divergenz der damit erfassten Phänomene erklären könnte? Gibt es doch nicht nur ganz unterschiedliche, vielfältige Formen der Hetero- und Homosexualität, sondern, wie ich im Folgenden mit Bezug auf empirische Studien zeigen möchte, auch erhebliche Unterschiede 
zwischen männlichen und weiblichen Homosexualitäten, wobei für Letztere ihre verblüffende Plastizität charakteristisch ist.

\section{Formen weiblicher Homosexualität}

Die separate Betrachtung von weiblicher Homosexualität bleibt nicht unwidersprochen. Le Soldat (2015) beispielsweise lehnt eine solche gesonderte Behandlung (S. 28) ab. Denn, so ihre Begründung, bei einer künstlichen Auftrennung in ein kategoriales Denken unter männlich und weiblich würden zentrale Einsichten verloren gehen (S. 29).

Rohde-Dachser (1994) vertritt einen anderen Standpunkt, dem ich mich anschliesse. Weibliche Homosexualität werde in den klassischen psychoanalytischen Theorien weitgehend ausgeblendet, Homosexualität mit männlicher Homosexualität gleichgesetzt (S. 829). Auch die Pathologisierung weiblicher Homosexualität sei ausgeprägter.

Weibliche Homosexualität unterliegt auf diese Weise einem noch stärkeren Trend zur Pathologisierung als die männliche Homosexualität, in der sich die narzißtische Objektwahl immerhin auch als ein Schritt der Befreiung aus der Beziehung mit dem Primärobjekt interpretieren läßt. (Rhode-Dachser, 1994, S. 832)

Zwar lassen sich die Spezifika der weiblichen Homosexualität nur im Vergleich mit der männlichen erkennen. Aber dann erweist sich, dass diese Unterschiede über die Differenz von Ähnlichem (Le Soldat, 2015, S. 28) hinausgehen, sodass sich bei der Darstellung und Auswertung dieser Besonderheiten der psychoanalytische Blick sinnvollerweise auch auf weibliche Homosexualität alleine konzentrieren sollte. Das mindert ausserdem die Gefahr, die weibliche Homosexualität doch wieder der männlichen - zu der es zahlreiche Arbeiten gibt anzuhängen oder unterzuordnen.

Weibliche homosexuelle Entwicklungen sind charakterisiert durch ihre Diversität und Heterogenität. Die sexuelle Orientierung - ich greife im Folgenden auch auf Ergebnisse aus Nachbardisziplinen $\mathrm{zu}^{7}$ - kann sich bei Frauen über die ganze Lebensspanne hinweg ändern, und das geschieht nicht selten sogar erst im mittleren Lebensalter, also bei Frauen, die lange in einer heterosexuellen Beziehung lebten und sich dann in eine Frau verlieben. Schon die Psychologin Düring (1994) macht in ihrer Arbeit Sequentielle Homo- und Heterosexualität diese Beobachtung, ebenso wie die US-amerikanischen Psychoanalytiker*innen Downey und Friedman 
(1998). Bei Frauen kann die emotionale, vielleicht zunächst freundschaftliche Bindung an eine andere Frau sexuelle Gefühle und Fantasien entfachen (S. 490), während bei Männern, so die Autor*innen, solch eine Weiterentwicklung oder ein Umschlag blosser Sympathie in Sexualität praktisch nicht vorkommt.

Die Sexualforscher Kinnish et al. (2004) fanden in ihrer retrospektiven Studie signifikante geschlechtsspezifische Differenzen hinsichtlich der Flexibilität der sexuellen Orientierung bei (homo-und heterosexuellen) Männern und Frauen. Die Autoren operationalisierten den Begriff der sexuellen Orientierung für ihre Studie, indem sie ihn in drei Faktoren unterteilten: sexuelles Verhalten, sexuelle Fantasien und romantische Attraktion (S. 29). In allen drei Dimensionen berichteten, ihrer Untersuchung zufolge, lesbische Frauen von häufigeren Wechseln als schwule Männer (S. 36). Die Autoren fanden «bei lesbischen Frauen ein Muster hoher Wechsel-Indizes, die mit dem Alter zunahmen, während schwule Männer niedrige Wechsel-Indizes hatten, die unabhängig vom Alter auf niedrigem Niveau stabil bleiben» (S. 36).

Im gegebenen Zusammenhang besonders bemerkenswert an den Ergebnissen dieser empirischen Untersuchung ist, dass knapp zwei Drittel aller Befragten von irgendeiner (wenn auch kleinen) Veränderung ihrer sexuellen Orientierung berichteten, wohingegen ein gutes Drittel des Samples keinerlei Veränderung erlebte - $33 \%$ der schwulen und 66 \% der heterosexuellen Männer, aber nur 9 \% der lesbischen und 51\% der heterosexuellen Frauen (S. 38). ${ }^{8}$

Die Gruppe der invariant lesbischen Frauen ist signifikant kleiner als die entsprechende Gruppe homosexueller Männer. Vermutlich deshalb ist diese Spielart von Homosexualität mehr im Hinblick auf Männer beschrieben worden: Freud nennt sie die absolut Invertierten; bei Kinsey sind das die Probanden auf der Stufe 6 (ausschliesslich homosexuell) und bei Le Soldat sind es diejenigen mit Grund zur Homosexualität.

Wegen der Diversität lesbischer Homosexualität macht es Sinn, auch theoretisch entsprechend zu differenzieren und verschiedene, eventuell sogar konträre Theorien zur weiblichen Homosexualität heranzuziehen.

\section{Der Lesbische Komplex - die Warum-Perspektive}

Ihr Konzept des Lesbischen Komplexes hat Poluda-Korte erstmals in der von Alves (1993) herausgegebenen Anthologie Stumme Liebe vorgestellt; die unter diesem Titel zusammen gefassten Arbeiten feministischer Autorinnen, überwiegend Psychoanalytikerinnen, spiegeln die damalige Bandbreite theoretischer Positionen zu weiblicher Homosexualität wider. 
Eine neue Vokabel, so nennt Alves, die Herausgeberin von Stumme Liebe, in ihrem Vorwort das von Poluda-Korte eingeführte Konzept des Lesbischen Komplexes. Poluda-Korte arbeitet einen frühen inneren Konflikt im Leben aller Mädchen heraus, den sie für konstitutiv im Hinblick auf ihre Weiblichkeitsentwicklung hält.

Das Schicksal des sexuellen Begehrens der Tochter ihrer Mutter gegenüber nennt die Psychoanalyse den «negativen weiblichen Ödipuskomplex». Der BegriffÖdipuskomplex istanschaulich, wenn er sich aufdas Begehren des Sohnes gegenüber seiner Mutter bezieht. Mit dem negativen Ödipuskomplex ist dessen Umkehrung gemeint, das Begehren des Sohnes seinem Vater gegenüber. Abgesehen davon, daß diese Benennung unanschaulich und schwer verständlich ist, suggeriert sie eine Bewertung, die bewußt nicht beabsichtigt war. Der Begriff positiver weiblicher Ödipuskomplex entspricht einer wiederum unanschaulichen Ableitung der weiblichen Entwicklung von der männlichen und betrifft die Liebe der Tochter zum Vater. (...) Der Ausdruck negativer weiblicher Ödipuskomplex schließlich bildet einen doppelten Widerstand gegen die Wahrnehmung dessen, was er benennen will. Deshalb möchte ich mich (...) von diesem verschleiernden sprachlichen Ballast befreien und in diesem Artikel treffender und anschaulicher vom «lesbischen Komplex» reden. (Poluda-Korte, 1993, S. 74)

Am Anfang der weiblichen psychosexuellen Entwicklung, führt die Autorin aus, steht die präödipale Beziehung zur Mutter, die Poluda-Korte nach Chodorow die matrisexuelle nennt.

Ich würde behaupten, dass während der matrisexuellen Dyade, in der das Leben im Körper der Mutter durch ein Leben auf dem Körper der Mutter abgelöst wird, eine konkrete, oral konditionierte Intimbeziehung zwischen Mutter und Kind besteht, als eine Spielart bzw. Urform des sexuellen Verkehrs von Form und Inhalt. (Poluda-Korte, 1996, S. 74)

Die Entwicklungskrise durch die fortschreitende Entdeckung der Welt und die zunehmende Separation von der Mutter, die Poluda-Korte in Anlehnung an Mahler auf das Ende des ersten Lebensjahres datiert, beschert dem Säugling 
eine im kleinianischen Sinne frühe ödipale Enttäuschung: Das kleine Kind muss akzeptieren, «daß das Liebespaar nicht länger Mutter und Kind ist, sondern daß der eigentliche Liebespartner der Mutter der Vater ist.» (Poluda-Korte, 1996, S. 74)

Es gibt, so die Autorin weiter, aber eine unterschiedliche Belastung der Geschlechter, die eine unterschiedliche Ökonomie der Wut bedingt: Junge und Mädchen müssen zwar gleichermassen auf alle Lustmodalitäten mit der Mutter verzichten lernen, aber das Mädchen muss, anders als der Junge, nicht nur erkennen, dass Mutter und Vater ein Paar sind, also die Generationsschranke und das Inzesttabu akzeptieren, sondern darüber hinaus verkraften, dass sie nie wieder Zugang zum Körper der Mutter/der Frau haben wird, wenn sie, wie es von ihr erwartet wird, in die heterosexuelle Verkehrsordnung (S. 75) eintritt. Diese doppelte Kränkung, «diese Verunsicherung durch die homosexuelle Enttäuschung an der Mutter ist der Kern des lesbischen Komplexes, durch den Liebe in Haß» (S. 75) umschlagen und zu einem tiefen Groll führen kann, der wiederum ein frühes, strenges Über-Ich auf den Plan ruft bzw. zu Selbstvorwürfen führt, die eigentlich dem untreuen Liebesobjekt, der Mutter gelten.

Die Qualität der frühen Beziehung entscheidet darüber, ob das Erleben dieser doppelten Zurückweisung durch die Mutter als unumstösslicherVerrat erlebt wird, der einen lebenslangen Groll hervorruft und dann u. U. in die Partnerschaft zu einem Mann einfliesst, oder ob die Liebe zu ihr als Treue und Loyalität bestehen bleibt. Die Identifizierung mit der anfangs heiss und homosexuell geliebten Mutter bildet die Basis der Weiblichkeit, des Begehrens und Begehrt-Werdens.

Später lesbische Mädchen, vermutet Poluda-Korte (1993),

... akzeptieren das Homosexualitäts-Tabu nicht, oder so halbherzig, daß sie dessen Akzeptanz in einem späteren "Coming out» wieder rückgängig machen, sie bestehen aufihrem primären Wunsch nach Frauenliebe und verweigern oder revidieren den Objektwechsel. Die Wut über die homosexuelle Zurückweisung der Mutter binden sie nicht ins Über-Ich, sondern halten sie zur Behauptung ihres Begehrens im Ich aufrecht und wetteifern mit dem Vater. (S. 95 f.)

Ähnlich Rohde-Dachser (1994):

Das so entstandene Phantasiemuster, in dem sich die erotisierte Beziehung zu einer Frau abbildet (...), kann später-grundsätzlich oder bei Bedarf - in eine homosexuelle Beziehung einmünden. Die 
autoerotische Betätigung des kleinen Mädchens, die eine solche homosexuelle Entwicklung einleitet oder doch vorbahnt, dient damit gleichzeitig der Überbrückung eines Beziehungsdefizits; sie steht im Dienst eines Beziehungswunsches (nach Nähe, nach körperlicher und emotionaler Intimität, nach Bindung) angesichts einer diese Wünsche ganz oder weitgehend versagenden Realität. (S. 838)

Die Revidierbarkeit des Objektwechsels und der Objektwahl, von der beide Autorinnen sprechen, hat das freudsche Modell der konstitutionellen Bisexualität im Sinne der unbewusst doppelten, homo- und heterosexuellen Objektwahl zur Voraussetzung. Denn nur so ist ein solches Wählen «im Dienst der Lust und im Dienst der Abwehr» (Rohde-Dachser, 1994, S. 827) denkbar. ${ }^{9}$

Poluda-Kortes und auch Rohde-Dachsers Konzeptualisierung eignet sich gut für das Verstehen der weitaus grössten Gruppe lesbischer Frauen, deren sexuelle Orientierung bis zu einem gewissen Grad über die ganze Lebensspanne hinweg oder in bestimmten Lebensphasen flexibel ist, sodass Freuds Grundannahme der Bisexualität in seiner Relevanz hier unmittelbar einleuchtet.

Behandlungstechnisch bedeutet das, dass die Behandler*in eine grösstmögliche Offenheit bzgl. der unbewussten Konflikthaftigkeit der sexuellen Orientierung im Sinne Rohde-Dachsers und Poluda-Kortes anstrebt und die Frage nach den Beweggründen für einen Wechsel der sexuellen Orientierung mit bedenkt - eine homosexuelle wie auch eine heterosexuelle Partner*in-Wahl kann neurotische Gründe haben. Ziel der Behandlung ist die Schaffung eines möglichst grossen Spielund Fantasieraums für die jeweils abgewehrte (homo- oder hetero-) sexuelle Seite.

Anna Koellreuter stellt eine Fallvignette zurVerfügung, die diese Offenheit demonstriert. Sie berichtet von einer 30-jährigen lesbischen Patientin, die ihr schon in den Vorgesprächen zwei Bedingungen stellt: Sie wolle das Du mit der Analytikerin, weil ihr ein Sie unter Frauen eigenartig vorkomme, und die Analytikerin müsse sie als Lesbe akzeptieren. Koellreuter lehnt die erste Bedingung zwar ab, sagt der Patientin aber, dass sie mit ihrem Lesbisch-Sein kein Problem habe. Die Analytikerin spürt dann während der Vorgespräche ein leichtes Unbehagen, das sie nicht recht zuordnen kann. Nach einer mit aktuellen, eher äusseren Problemen gefüllten ersten Analysezeit breitet sich eine zunehmende Lähmung in den Analysesitzungen aus. Koellreuter vermutet allmählich, dass ihre schnelle Akzeptanz der Homosexualität ihrer Patientin der Grund für das Stagnieren des analytischen Prozesses sein könnte. 
Denn ich hatte ihr auf unanalytische Weise vermittelt, ich fände es gut, daß sie Lesbe sei-und ihr dadurch ein offenes Suchen verbaut. Ich realisierte, daß ich ihr den Raum für eine mögliche andere Lebensweise damit genommen hatte, so dass sie mir permanent ihr Lesbischsein beweisen mußte. (Koellreuter, 2000, S. 137)

Durch ihre Gegenübertragungsanalyse stellt Koellreuter fest, dass sie Angst vor möglichen Liebesangeboten der Patientin, also eine eigene abgewehrte Triebangst hat. Ihr Statement zu Beginn, sie habe mit Lesben keine Probleme, versteht sie im Nachhinein als Versuch, die Differenz zwischen ihr und ihrer Patientin aufzuheben. Sie entscheidet sich,

ihr zu sagen: «Sie möchten mir seit der ersten Stunde beweisen, daß Sie eine überzeugte Lesbe sind, aber eigentlich spielt es für mich keine Rolle, ob Sie Lesbe oder Hetera sind. Wir machen zusammen eine Analyse, und darin ist offen, was geschieht." Anschließend entstand eine heftige Diskussion über die Abwertung der Lesben durch die Heteras, wie ich eben eine war - aber in der Folge löste sich die Sprachlosigkeit langsam auf, entspannte sich unser Verhältnis zusehends. Die Analyse nahm ihren Lauf. (Koellreuter, 2000, S. 138)

Diese Intervention Koellreuters ist ein erfolgreicher Versuch, den analytischen Raum zu erweitern. Er zeigt, wie die Analytikerin, ausgehend von der These der unbewussten Konflikthaftigkeit sexueller Objektwahl, ihre Patientin nicht in deren vermeintlich unveränderlichen Homosexualität belässt. Ein vorbehaltloses Akzeptieren der Homosexualität ihrer Patientin wäre gemäss Koellreuters Selbstreflexion unanalytisch gewesen.

Diese der Patientin auch einsehbar gemachte Öffnung hat konzeptionell die freudsche konstitutionelle Bisexualität zur Prämisse, weil nur so die Offenheit der Objektwahl analytisch gedacht werden kann; letztlich lässt sich Koellreuter also von der Warum-Frage und der von diesem Ansatz her gewonnenen Überzeugung leiten, dass sexuelle Orientierung aus unbewussten inneren Konflikten resultiert.

\section{Basic facts of homosexual life - die Wie-Perspektive}

Statt auf das Warum richtet Ermann (2009) sein Augenmerk auf das Wie homosexueller Entwicklungen und verfolgt in seinem Artikel Das homosexuelle Dilemma - Die Entwicklung der normalen männlichen Homosexualität diese 
Fragestellung anhand des Arbeitsbegriffs basic facts of homosexual life (S. 359). Er ergänzt damit die von Money-Kyrle formulierten facts of life $e^{10}$ um seiner Meinung nach unausweichliche existenzielle Erfahrungen ${ }^{11}$, die ein homosexueller Junge bewältigen muss: Durch die fehlende homosexuelle Spiegelung durch einen heterosexuellen Vater gerät der Junge in das homosexuelle Dilemma (S. 356) - er muss sich zwischen den Entwürfen, die ihm seine heterosexuellen Objekte zuschreiben, und dem eigenen, u. U. schon vor der Geburt angelegten Präferenzschema für die spätere Geschlechtspartnerwahl entscheiden. Dabei geht Ermann (2009) davon aus, dass sich die Dimensionen Kerngeschlechtsidentität, Geschlechtsrollenidentität und sexuelle Orientierung verschränkt und voneinander untrennbar entwickeln (S. 356), so wie Reiche (2007) von der Gleichursprünglichkeit von Kerngeschlechtsidentität und Objektwahl spricht (S. 937).

Später erlebt der - im Sinne eines pränatalen Präferenzschemas - homosexuelle Junge eine spezielle ödipale Situation, für die Ermann den Ausdruck homosexuelle Triade (S. 357) vorschlägt, weil sie sich nicht mit der heterosexuellen Urszene der ödipalen Situation gleichsetzen lässt: Der Junge begehrt

\section{(...) im Vater einen Mann, der ihn zurückweist, indem dieser Frauen liebt. Dieser Vater muss ihm die ersehnte Glückseligkeit und Bestätigung seiner sexuellen Identität schuldig bleiben. In der Zurückweisung wiederholt sich das homosexuelle Dilemma in einer sexuellen Dimension. (...) Es gibt keine homosexuelle Urszene, mit der er sich identifizieren kann. (Ermann, 2009, S. 358)}

Dieses Ausbleiben der Spiegelung einer homosexuellen Identität durch einen heterosexuellen Vater - Ermann verwendet einen Begriff aus der Mentalisierungsforschung und spricht von nicht kontingenteri.S. von nicht kongruenter Spiegelung bzw. von fundamentaler Diskontingenz (S. 355) - betrachtet der Autor als Kernkomplex und als Organisator der weiteren homosexuellen Entwicklung (S. 354). Um eine gesunde homosexuelle Identität zu entwickeln, muss der Junge «sich von ‘falschen heterosexuellen Lebensentwürfen (...) entidentifizieren und eine «ungedeckte` Identität (...) entwickeln» (S. 359). Ich verstehe Ermann so, dass auch eine bewusst noch so resonante Haltung eines heterosexuellen Vaters seinem homosexuellen Jungen gegenüber dieses Dilemma höchstens mildern, nicht aber aufheben kann, weil die jeweiligen Urszenenfantasien nicht deckungsgleich sind. ${ }^{12}$ 
Ermanns Überlegungen folgen der Wie-Frage. Mit der Herausarbeitung von spezifischen inneren Konfliktsituationen - der basic facts of homosexual life: dem homosexuellen Dilemma und der homosexuellen Triade, die also Folge homosexueller Entwicklung sind, und nicht deren Ursache - setzt der Autor neue und klinisch relevante Impulse.

Die Übertragbarkeit der von Ermann nur für die männliche homosexuelle Entwicklung formulierten Thesen auf lesbische Mädchen stützt sich z. B. auf die nonkonformen Geschlechtsrollenerfahrungen, die bei Jungen als starker Prädiktor für eine später manifest werdende homosexuelle Orientierung ${ }^{13}$ gelten. Diese Geschlechts-Nonkonformität zeigen die von Düring (1993) sogenannten wilden Mädchen, zu denen sie geforscht hat. Ihr zufolge bevorzugen wilde Mädchen statt Puppen, mit denen sie wenig anzufangen wissen, aufregendere rough-and-tumblegames. Zwar werden keineswegs alle wilden Mädchen lesbisch, aber in der Biografie vieler in Bezug auf ihre Geschlechtsrollenpräferenz maskulin orientierten Lesben finden sich diese nonkonformen Geschlechtsrollenerfahrungen.

Soweit ich sehe, gibt es auffällig wenige ausführliche und zeitgenössische Kasuistiken lesbischer Frauen. Trifft das zu, so steht das im eklatanten Gegensatz zu der Vielzahl entsprechender Fall-Darstellungen homosexueller Männer, z.B. publiziert von Galatzer-Levy (2001), Phillips (2001), Reiche (2007), Ermann (2009), Roughton (2012).

Umso wichtiger ist daher die Analyse, die Mechthild Zeul in Alves' Reader Stumme Liebe (1993) veröffentlicht hat. An ihrer Kasuistik lässt sich sowohl die Relevanz der Warum- als auch die der Wie-Frage demonstrieren.

Zeuls Analysandin ist Stewardess und seit drei Jahren mit einer Frau zusammen. Sie leidet unter schweren depressiven Verstimmungen und Wutausbrüchen der Freundin gegenüber sowie unter starken Flugängsten. Die Patientin, in beengten Verhältnissen aufgewachsen, hatte mit einem ihrer Brüder lange im Bett der Eltern geschlafen - dort sei «nachts die Post abgegangen». Diese Art Belagerung und Überstimulierung wiederholt sich auch in der Übertragung, ausgelöst durch eine Begegnungsszene unmittelbar vor der ersten Analysestunde: Die Analytikerin eilt mit Einkaufstaschen bepackt in ihre Praxis, vor der die Patientin bereits auf sie wartet. Die Patientin befürchtet nun, wie sie ihrer Analytikerin nach einer Weile anvertrauen wird, dass diese durch nächtliche Liebesaktivitäten und die Versorgung der vielen männlichen Familienmitglieder zu erschöpft sei, um sich mit ihr wirklich zu befassen, dass, um im Bild der Patientin zu bleiben, bei der Analytikerin die Post abgegangen sei. Die Patientin aber braucht ihre Analytikerin zunächst unbedingt als Frau, die unbelastet und frei für sie ist. Von dieser selbstständigen, schönen 
und klugen Analytikerin-Mutter ist die Patientin sexuell angezogen und versucht, sie durch Geschenke, durch Exotisches, das sie von ihren Flugreisen mitbringt, zu gewinnen. Zeul nimmt diese Geschenke an («immer traf sie meinen Geschmack», S. 170); sie geht davon aus, dass die Patientin «dieses Stück guter, heiler Beziehung» (S. 170) braucht, um sich mit ihrer Aggression auseinandersetzen zu können, die sich insbesondere in ihrer Partnerschaft entlädt, bis hin zu Handgreiflichkeiten. Die Bearbeitung dieser Beziehungsdynamik erweist sich als schwierig, denn die Patientin wirft ihrer Analytikerin vor, sie, wie alle Psychoanalytiker und der «Frauenfeind Freud», von der Homosexualität und der Freundin wegbringen und in die Arme von Männern treiben zu wollen.

In dieser schwierigen Zeit wirkt die Patientin, die zwar mitteilt, wenn sie berufsbedingt fehlt, ihrer Analytikerin aber vorenthält, wo sie unterwegs ist, «wie vom Erdboden verschluckt», so Zeuls Erleben in der Gegenübertragung. Es stellt sich allmählich heraus, was die Patientin vor ihrer Analytikerin verbergen will: Im Erleben der Patientin ist das Fliegen mit sexuellen Abenteuern und bisher verpönten Fantasien verbunden, nämlich der, sie drängele, auch beim Sex, wie ein Mann, und es werde herauskommen, dass sie sich unter Vorspiegelung der falschen Tatsache, sie sei eine Frau, bei der Fluggesellschaft beworben habe.

Diese Fantasien, Ängste und Wünsche ihrer Patientin nennt Zeul heterosexuell. Das ist ein bisschen verwirrend, weil diese Wünsche beim Lesen der Kasuistik nicht heterosexuell erscheinen, sondern offenbar den Wunsch der Patientin zeigen, die männliche Position beim Geschlechtsverkehr mit der Freundin zu übernehmen (S. 184), triebtheoretisch gesprochen, die aktive Position einnehmen zu wollen. Dieses Mehr an Spielraum in Richtung Maskulinität zulassen zu können, versteht Zeul als Erweiterung des psychischen Spielraums ihrer Patientin. Die Patientin selbst hat jetzt auch keine Angst mehr, eigentlich heterosexuell zu sein oder sich in der Beziehung mit der Freundin etwas vorgemacht zu haben; sie kann sich psychisch weiter vorwagen und ihren Fantasien Raum geben.

«Erst jetzt liess sie Phantasien zu, die sich um meine Beziehung zu einem Mann drehten, ohne darüber in Wut zu geraten.» (Zeul, 1993, S. 182) Als ihre Analytikerin aber die Praxis in ihre Wohnung verlegt, steigt die Angst wieder und die Patientin fürchtet sich, so Zeul,

vor ungemachten Betten und vor meiner Sexualität mit einem Mann. Vielleicht - so die Patientin - vernachlässigte ich eines Mannes wegen meine intellektuellen und eigenständigen Interessen und Aktivitäten; vielleicht sei ich eine Hure wie die Mutter, die 
es mit Männern trieb und darüber ihren Intellekt vergaß. (Zeul, 1993, S.182)

Zeul beschreibt, wie sie es zulässt, von der Patientin verführt zu werden und sie als schöne Frau wahrzunehmen, von der versorgt zu werden angenehm sein müsse. Diese Offenheit gibt der Patientin die Möglichkeit, ihrer Analytikerin die Beziehung mit Männern und sich selbst männliche Seiten zuzugestehen. So erzählt sie von ihrem Kater, der nachts um die Häuser streife und seinen Samen verteile.

Zugleich aber stabilisierte sich in dieser Zeit die Beziehung, (...) weil die Patientin angst- und schuldfrei ihre sexuellen Wünsche äußerte und die Freundin darauf einging. (...) Wir erarbeiteten, dass sie sich der Freundin durchaus sexuell, wie ein Mann, nähern konnte, ohne dabei ihre Weiblichkeit zu verlieren. (Zeul, 1993, S.185)

Zeul nimmt die Liebesübertragung der Patientin an ${ }^{14}$ und ermöglicht ihr auf diese Weise, ihr sexuelles Potenzial besser auszuschöpfen. Die Akzeptanz und das Ausleben der maskulinen Seiten der Patientin waren vor der Analyse wegen des Misstrauens ihren eigenen Impulsen gegenüber und wegen der ständig drohenden Verwechslung von Maskulinität mit Verlust von Weiblichkeit gehemmt gewesen.

Zeul legt den Akzent nicht auf die Psychogenese der Homosexualität ihrer Patientin, nicht auf das Warum. Beim Lesen der Kasuistik entsteht aber der Eindruck, dass sie die lesbische Objektwahl ihrer Patientin als progressive Lösung in einem unbewussten inneren Konflikt versteht, der von traumatischen Urszenenerlebnissen, von Überstimulierung im beengten Bett der Eltern aufgeheizt worden war. Möglicherweise, das wäre meine Vermutung, gab die Patientin vor diesem biografischen Hintergrund zur Bewältigung ihrer eigenen Triebhaftigkeit unbewusst aktiven Triebzielen den Vorrang, verdrängte dann aber die entsprechenden Fantasien, die sie insgeheim als unweiblich, verwirrend und kompromittierend erlebte. Das machte ihre Neurose aus. ${ }^{15}$ Die Anerkennung ihrer Wünsche, sich der Freundin wie ein Mann zu nähern, so beschreibt es Zeul, brachte Erleichterung und ein Mehr an sexuellem Spielraum. Vielleicht konnte Zeuls Patientin diese Maskulinität in der Beziehung zu einer Frau besser unterbringen als in der Beziehung zu einem Mann. ${ }^{16}$ Das sind, bezogen auf diesen Fall, Überlegungen, die sich von der Warum-Frage herleiten. 
Gleichzeitig handelt es sich bei der Patientin um eine stabile lesbische Orientierung, was Zeul nicht nur zu keinem Zeitpunkt in Frage stellt; sie begleitet vielmehr ihre Patientin auf dem Weg hin zu mehr Maskulinität. Insofern ist diese Kasuistik auch ein Beispiel für die Anerkennung der sexuellen Identität und die Arbeit am Wie lesbischer Persönlichkeitsentwicklung. Bei Zeuls Patientin könnte es beispielsweise eben diese Maskulinität sein, die sie bisher verdrängen musste, weil es für diese nonkonforme Geschlechtsrollenidentität keine bestätigende (bzw. kontingente sensu Ermann) Spiegelung durch ihre Mutter gegeben hatte - eine Narbe oder Wunde, die immer wieder zu Problemen führen musste.

Zeuls Kasuistik demonstriert also den Fall, für den sowohl die Warum- als auch die Wie-Frage fruchtbar ist. Es fehlt nun ein drittes Beispiel für die vorrangige oder sogar ausschliessliche Anwendbarkeit des Wie-Konzepts in der Behandlung einer lesbischen Frau, deren Homosexualität nicht das Ergebnis eines unbewussten inneren Konflikts, sondern konstitutionell bedingt ist und sie in ihrer psychosexuellen Entwicklung mit den facts of homosexual life konfrontieren wird. Ausführliche Kasuistiken lesbischer Frauen von zeitgenössischen Analytikerinnen zu finden, erwies sich bei meinen Recherchen ohnehin als schwierig, für diesen speziellen Fall aber als unmöglich. Das vermutlich aus zwei Gründen: Weibliche sexuelle Orientierung ist flexibler, variantenreicher und «gestreuter» (z. B. auf der Kinsey-Skala) als die von Männern (siehe z. B. Anmerkung 8), und es ist deshalb zu erwarten, dass es relativ gesehen sehr viel weniger Frauen als Männer gibt, die konstitutionell bedingt lesbisch sind. Auswirkung hat aber auch eine Art psychoanalytischer lag-die erheblich zeitversetzt einsetzende Beschäftigung mit der (Homo) Sexualität von Frauen, welche die Zahl der Kasuistiken lesbischer Patientinnen zusätzlich reduziert.

\section{Schlussbetrachtung}

Wenn alle der vielen Spielarten weiblicher Homosexualität Beachtung finden sollen, dann auch die der Frauen mit Grund zur Homosexualität wegen einer inneren Notwendigkeit (Le Soldat) oder einer genetischen oder endokrinologischen Mitbeeinflussung (Ermann), also die Frauen, die bisher in psychoanalytischer Theorie und entsprechend auch Praxis wenig Raum hatten.

Die hier vorgestellten Konzepte der konstitutionellen Mit-Bedingtheit von Homosexualität und der psychodynamischen Entwicklung von Homosexualität sind ebenso heterogen wie plausibel. Daher kommt sowohl in theoretischer wie auch in klinischer Hinsicht der Rückgriff auf eines jedes von ihnen bei der Betrachtung weiblicher Homosexualität in Frage. Sie haben jeweils ihre spezifi- 
schen Vorteile: Die Fokussierung auf das Wie der homosexuellen Entwicklungen zeichnet sich durch besondere Anschaulichkeit aus, stellt die lesbische Objektwahl nicht in Frage und kann damit den Boden schaffen für eine intensive Arbeit an inneren Konflikten - unabhängig davon, ob diese nach Ermann in der Folge einer homosexuellen Orientierung oder anderweitig entstehen. Für die Fokussierung auf das Warum eignen sich abstraktere Modelle, z. B. das der Bisexualität, wie PoludaKorte das vorgeschlagen hat, und zwar sowohl im Sinne einer konstitutionell angelegten (bisexuellen) Möglichkeit zur männlichen und weiblichen Objektwahl, als auch im Sinne einer konstitutionell angelegten, bisexuellen - im Sinne gleichzeitig vorhandener femininer und maskuliner - Position in der Sexualität. Die Frage nach der psychogenetischen Entstehung der sexuellen Orientierung ist weiterhin hochrelevant (Dannecker, 2000, Koellreuter, 2000, Reiche, 2007) und hält, das ist ein weiterer Vorteil, bei der sehr grossen Gruppe der sequenziell lesbischen Frauen die Tür in Richtung einer neuen, nicht-neurotischen Partner*in-Wahl über einen längeren Zeitraum offen.

Für die weit gefächerten weiblichen Homosexualitäten steht also dieser (oder in Zukunft ein vielleicht noch viel grösserer?) Fächer an Theorien bereit.

\section{Literatur}

Alves, E. (Hrsg.) (1993). Stumme Liebe. Der «lesbische Komplex» in der Psychoanalyse. Freiburg i. Br: Kore Verlag.

Castendyk, S. (1998). Vom «Objektwechsel» zur Objektwahl - Die Psychoanalyse als Grundlage eines nicht pathologischen Verständnisses der weiblichen Homosexualität. In: Texte aus dem Colloquium Psychoanalyse. Jg. 2, Heft 3, 90-106.

Dannecker, M. (2000). Probleme der männlichen homosexuellen Entwicklung. In: Psyche-Z Psychoanal 54. 1251-1277.

Downey, J. \& Friedman, R. (1998). Female homosexuality: classical psychoanalytic theory reconsidered. J Am Psychoanal Assoc 46 (2). 471-506.

Düring, S. (1993). Wilde und andere Mädchen. Die Pubertät. Freiburg i. Br.: Kore-Verlag.

Düring, S. (1994). Über sequentielle Homo- und Heterosexualität. Z Sexualforsch. Bd. 7. 193-202.

Ermann, M. (2009). Das homosexuelle Dilemma. Zur Entwicklungsdynamik der normalen männlichen Homosexualität. Forum Psychoanal 25. 249-261.

Galatzer-Levy, R. (2001). Finding our way in perplexity: the meaning of sex in the analysis of a gay man. J Am Psychoanal Assoc 49/4. 1219-1233. 
Godfrind, J. (2000). Die weibliche Homosexualität in der analytischen Kur. In: Heenen-Wolff (Hrsg.) Neues vom Weib. Französische Beiträge. Psychoanalytische Blätter Bd. 16. Göttingen: Vandenhoeck \& Ruprecht. 53-81.

Gsell, M. \& Zürcher, M. (2011). Licht ins Dunkel der Bisexualität. Bisexualität, anatomische Geschlechtsdifferenz und die psychoanalytische Bedeutung von männlich und weiblich. Psyche - Z Psychoanal 65. 699-729.

Kinnish, K., Strassberg, D. \& Turner, C. (2004). Geschlechtsspezifische Differenzen der Flexibilität der sexuellen Orientierung. Eine mehrdimensionale retrospektive Studie. Z Sexualforsch 2004/ 17. 26-45.

Koellreuter, A. (2000). Das Tabu des Begehrens. Zur Verflüchtigung des Sexuellen in Theorie und Praxis der feministischen Psychoanalyse. Giessen: Psychosozial-Verlag.

Kreische, R. (2005). Homosexualität: Angst vor Fremdem. http:/ / mobile.aerzteblatt. $\mathrm{de} /$ print/45873.htm (01.08.15, 08:20).

Le Soldat, J. (2015). Grund zur Homosexualität. Vorlesungen zu einer neuen psychoanalytischen Theorie der Homosexualität. Stuttgart-Bad Canstatt: fromann-holzboog.

Phillips, S. (2001). The overstimulation of everyday life: I. New aspects of male homosexuality. J Am Psychoanal Assoc 49/4. 1235-1268.

Poluda-Korte, E. (1993). Der «Lesbische Komplex». Das homosexuelle Tabu und die Weiblichkeit. In: Alves E. (Hrsg.) (1993). Stumme Liebe. Der «lesbische Komplex» in der Psychoanalyse. Freiburg i. Br.: Kore Verlag. 73-132.

Poluda-(Korte), E. (1996). Die Rolle des Homosexualitätstabus für die weibliche Entwicklung. In: Grosz-Ganzoni, I. Widerspenstige Wechselwirkungen. Tübingen: edition discord. 65-84.

Rauchfleisch, U. (1993). Homosexualität und psychoanalytische Ausbildung. Forum Psychoanal 9/4: 339-347.

Reiche, R. (2007). Gender ohne Sex. In: Psyche - Z Psychoanal 51. 926-957.

Rohde-Dachser, C. (1994). Männliche und weibliche Homosexualität. In: Psyche-Z Psychoanal 48. 827-841.

Roughton, R. (2001). Rethinking homosexuality. What it teaches us about psychoanalysis. http://www.shrink-friendly.co.il/tau/article/Rethinking_homosexuality.pdf. (01.08.15, 08:30).

Roughton, R. (2012). Four men in treatment - an evolving perspective on homosexuality and bisexuality, 1965 to 2000. JAm Psychoanal Assoc 49/4. 1187-1217. 
Stakelbeck, F. \& Frank, U. (2006). Kommen die neuen psychoanalytischen Theorien zur männlichen Homosexualität nur noch aus Amerika? In: Biechele, U., Hammelstein, Ph. \& Heinrich, Th. (Hrsg.): Anders ver-rückt?! Jahrbuch Lesben - Schwule - Psychologie 2006. Lengerich: Pabst. 121-137.

Zeul, M. (1993). Klinische Anmerkungen zur weiblichen Homosexualität. In: Alves,

E. M. (Hrsg.) Stumme Liebe, Freiburg i. Br.: Kore Verlag. 163-188.

\section{Anmerkungen}

1 Vgl. z. B. Dannecker (2000, S. 1255 f.).

2 Nur sehr vereinzelt waren Stellungnahmen zu finden, z. B. Kreische (2005) im Ärzteblatt.

3 Von einer ähnlichen Dynamik berichtet Roughton (2001, S. 5).

4 Zur Geschichte von psychoanalytischen Institutionen und Homosexualität siehe z. B. Rauchfleisch (1993), Roughton (2001) oder Stakelbeck \& Frank (2006); zur z.T. widersprüchlichen Haltung Freuds siehe ebenfalls Roughton (2001).

5 Ich danke Herbert Will für diesen Hinweis und auch der von ihm geleiteten Schreibwerkstatt für deren Anregungen.

6 Roughton (2001) gibt einen Überblick über Arbeiten zu lesbischen und schwulen psychosexuellen Entwicklungen. Vgl. auch Stakelbeck \& Frank (2006) mit ihrem Aufsatz «Kommen die neuen psychoanalytischen Theorien zur männlichen Homosexualität nur noch aus Amerika?».

$7 \quad$ Vgl. auch Reiche (2007), der davon ausgeht, dass die Psychoanalyse keine vollständige Theorie der Geschlechtsidentität schaffen kann (S. 932).

8 Die Forschergruppe vermutet, dass dieser signifikante Geschlechtsunterschied bzgl. der Flexibilität der sexuellen Orientierung mit unterschiedlichen Sozialisationserfahrungen von Jungen und Mädchen zusammenhängen: Mädchen lernten Sexualität im Kontext sozialer Beziehungen; diese «stärkere Sensibilität für kontextuelle Einflüsse könnte bei Frauen zu größeren Schwankungen in der sexuellen Orientierung über die Lebensdauer führen» (S. $37)$.

9 Vgl. auch die interessanten Überlegungen von Castendyk (1998) zur lesbisch-psychosexuellen Entwicklung. Die Autorin geht davon aus, dass das Mädchen, nachdem es die Kränkung verarbeitet hat, nur ein Geschlecht verkörpern und im erotischen Dialog nur eine Position beziehen zu können (S. 100), durch eine homosexuelle Objektwahl «die sich stellenden Schwierigkeiten wirklich positiv gelöst» (S. 105) hat:Verschiedene Varianten sind denkbar, z. B. die Identifikation mit einem Vater, der ihr die Einlösung ihrer Lust am ehesten verspricht, oder, als Folge der Objektwahl, der Versuch, einem inzestuösen Kurzschluss durch das Ausweichen auf ein penisloses Geschöpf auszuweichen. «Beide Varianten, butch und femme, sind hier das Ergebnis einer Strategie, die besondere Konflikte jeweils entweder in der Positionierungsphase oder in der Phase der Objektwahl erfolgreich löst» (S. 105).

10 In Ergänzung von Money-Kyrles facts of life. Für Ermann (2009) geht es um «die Anerkennung dieses Dilemmas als Bedingung der homosexuellen Existenz. Es rückt damit für die homosexuelle Entwicklung in eine Reihe mit den drei Grundtatsachen, die Money-Kyrle (1968/71) als «basic facts of life» benannt hat: Getrenntheit und Abhängigkeit vom Anderen, Ausschluss von der elterlichen Urszene sowie Älterwerden und Tod» (S. 359).

11 Von solchen Erfahrungen handelt auch der Artikel The Overstimulation OfEvery Day des US-amerikanischen Psychoanalytikers Phillips (2001). 
12 Sehr interessant und anregend sind auch die Überlegungen Danneckers (2000): «Zwar läßt sich in psychoanalytischer Perspektive die Strukturierung des sexuellen Begehrens ohne die Einflüsse der Elternfiguren und die Interaktionen der realen Eltern mit dem Kind nicht denken. Aber diese Einflüsse sind nur die eine, in der psychoanalytischen Sexualitäts- und Homosexualitätstheorie allerdings fast ausschließlich verhandelte Seite. Die andere, nicht weniger wichtige Seite der Entwicklung betrifft den Einfluß einer im Kind bereits angelegten Struktur des Begehrens und der mit dieser einhergehenden sexuellen Phantasien und Wünsche auf die Interaktionen mit den Eltern. Von Freud wurde die Positionierung des Subjekts während seiner Entwicklung noch mit unhintergehbaren konstitutionellen Faktoren zusammengedacht. Und seine Annahme einer konstitutionellen Bisexualität hat sowohl seinem Konzept der Homosexualität als auch seiner Fassung des Ödipuskomplexes eine ganz bestimmte Kontur verliehen. Entwicklung wurde bei Freud nicht allein durch Entwicklung vorangetrieben, und es waren folglich auch nicht ausschließlich die Beziehungen des Kindes zu seinen Eltern, aus denen heraus die Struktur eines Subjekts abgeleitet wurde. Die sexuelle Struktur eines Subjekts wurde in derTheorie von Freud immer auch von den der Eltern-KindInteraktion vorgängigen konstitutionellen Faktoren beeinflußt» (S. 1260 f.).

13 Dannecker (2000) spricht vom homosexuellen, manchmal auch vom prähomosexuellen Jungen, Ermann (2009) von rudimentärer homosexueller Identität (S. 355).

14 Vgl. auch Godfrind (2000), die von Beendigungsphasen in Analysen berichtet, in denen ihr das nicht ganz gelungen ist, vielleicht auch nicht gelingen konnte.

15 Zur Schwierigkeit, die eigene Maskulinität zu integrieren, vgl. auch Castendyk (1998, S. 93): «eine Frau kann Mathematik studieren oder ein Flugzeug steuern, ohne das Gefühl zu haben, männlich zu agieren, aber kann sie auch mit derselben Sicherheit beim Paartanz führen oder im Liebesspiel penetrieren? Wohl kaum.»

16 Vgl. auch Castendyk (1998, S. 104): «Obwohl erotisch männlich positioniert, ist es (...) dennoch denkbar, dass das Mädchen eine heterosexuelle Objektwahl trifft; der von ihr später gewählte Mann wird dann, wenn die Beziehung glücklich verlaufen soll, weiblich positioniert sein. Wahrscheinlicher aber ist, dass die männliche Positionierung zu einer weiblichen Objektwahl führt: Als erwachsene Frau wird sie sich in weiblich positionierte Frauen verlieben und damit - ohne nennenswerte psychische Schwierigkeiten - glücklich werden.»

\section{Anmerkung zur Autorin}

Almut Rudolf-Petersen, Dipl. Psych., ist Mitglied der Deutschen Psychoanalytischen Gesellschaft (DPG) und der Deutschen Gesellschaft für Psychoanalyse, Psychotherapie, Psychosomatik und Tiefenpsychologie (DGPT) und Dozentin, Lehranalytikerin und Ausbildungsleiterin am DPG-Institut Hamburg. 